\title{
Stromal Fibroblasts in Digestive Cancer
}

\author{
Daniel L. Worthley • Andrew S. Giraud • \\ Timothy C. Wang
}

Received: 28 October 2009 /Accepted: 21 December 2009/Published online: 12 February 2010

(C) The Author(s) 2010. This article is published with open access at Springerlink.com

\begin{abstract}
The normal gastrointestinal stroma consists of extra-cellular matrix and a community of stromal cells including fibroblasts, myofibroblasts, smooth muscle cells, pericytes, endothelium and inflammatory cells. $\alpha$-smooth muscle actin $(\alpha$-SMA) positive stromal fibroblasts, often referred to as myofibroblasts or activated fibroblasts, are critical in the development of digestive cancer and help to create an environment that is permissive of tumor growth, angiogenesis and invasion. This review focusses on the contribution of activated fibroblasts in carcinogenesis and where possible directly applies this to, and draws on examples from, gastrointestinal cancer. In particular, the review expands on the definition, types and origins of
\end{abstract}

Sources of support: The Royal Australasian College of Physicians Cottrell Fellowship (D.L.W.) and the Queensland State Government, Smart State PhD award (D.L.W).

\section{L. Worthley}

Royal Brisbane and Women's Hospital Research Foundation

Clinical Research Centre,

Brisbane, Queensland, Australia

D. L. Worthley

Conjoint Gastroenterology Laboratory,

Queensland Institute of Medical Research,

Brisbane, Queensland, Australia

\section{A. S. Giraud}

Murdoch Children's Research Institute,

Royal Children's Hospital,

Melbourne, Victoria, Australia

T. C. Wang $(\square)$

Division of Liver and Digestive Diseases,

Department of Medicine, Columbia University Medical Center,

1130 St. Nicholas Avenue, Room 923,

New York, NY 10032, USA

e-mail: tcw21@columbia.edu activated fibroblasts. It examines the molecular biology of stromal fibroblasts and their contribution to the peritumoral microenvironment and concludes by exploring some of the potential clinical applications of this exciting branch of cancer research. Understanding the origin and biology of activated fibroblasts will help in the development of an integrated epithelial-stromal sequence to cancer that will ultimately inform cancer pathogenesis, natural history and future therapeutics.

Keywords Fibroblasts · Neoplasms · Digestive system neoplasms $\cdot$ Cancer stroma $\cdot$ Bone-marrow derived cells

\section{Introduction}

Digestive cancers cause a staggering global health burden [1]. In the US in 2008 it was estimated that more than 270,000 patients were diagnosed with and more than 135,000 died from cancers of the digestive system [2]. Carcinogenesis research has largely focused on the stepwise accumulation of molecular events within epithelial cells, but it is now clear that the peritumoural stroma is also critical in the initiation and progression of gastrointestinal cancer [3-9].

The normal gastrointestinal stroma consists of a structural and functional scaffolding known as the extra-cellular matrix (ECM) that supports an integrated community of stromal cells, including fibroblasts, myofibroblasts, smooth muscle cells, pericytes, endothelium and a variable population of inflammatory cells [8]. In addition, a specialized matrix known as the basement membrane separates the epithelium from the underlying stroma $[10,11]$. The predominant structural protein in the ECM is collagen, with type IV collagen the chief component of the basement membrane [9]. Other important ECM and basement 
membrane molecules include laminins, nidogen/entactin, proteoglycans such as perlecan and also fibronectin and tenascin [9].

Stromal cells communicate through direct cellular connections, paracrine mediators and cellular-ECM interactions [3]. Together, the basement membrane and the ECM are important in maintaining normal epithelial function and retarding carcinogenesis [9], as well as regulating stromal cell motility and migration $[7,12,13]$. In cancer, matrix metalloproteinases are important in ECM and basement membrane-remodelling and can promote carcinogenesis [9].

Invasive carcinoma is defined by the interruption of normal cellular compartments $[3,4,14,15]$. The invasion field between pre-existing epithelial and stromal compartments is a critical interface in carcinogenesis and leads to the important question of which compartment leads in this danse macabre [5]. Ultimately, however, one should address all aspects of cancer's heterotypic cellular biology, including malignancy, mesenchyme and matrix [14, 16-18].

This review focusses on the contribution of stromal fibroblasts to cancer and where possible directly applies this to, and draws on examples from, gastrointestinal carcinogenesis. In particular the review expands on the definition, types and origins of stromal fibroblasts. It examines their molecular biology and contribution to the peritumoral microenvironment and concludes by exploring some of the potential clinical applications of this exciting branch of cancer research.

The analogy of epithelium and stroma sharing a neoplastic "seed and soil" relationship was first documented 120 years ago [19]. Whilst, much of the molecular revolution has focussed on the epithelial "seed", there has been burgeoning interest in dissecting out the stromal factors that serve to initiate, support and progress carcinogenesis. Furthermore, it is likely that the stroma is more than simply a permissive accomplice, as suggested by the analogy, but rather will prove to be an active partner in carcinogenesis [20].

\section{Stromal Cells in Digestive Cancers}

More than half the mass of digestive cancers is usually accounted for by the cancer stroma [6]. Whilst gastrointestinal cancer stroma varies with cancer site and biology, as demonstrated by the specific lymphocytic stromal infiltrate found in hereditary non-polyposis colorectal cancer [21], many stromal events are common across different digestive cancers, solid organ cancers more generally and even some hematological malignancies [3]. In this section the main stromal cell types will be defined and discussed with special attention given to stromal fibroblasts and myofibroblasts.
Fibroblasts and Myofibroblasts

Fibroblasts and myofibroblasts are important cells within the tumor stroma. On light microscopy, fibroblasts appear as spindle shaped cells surrounded by ECM. In normal gastrointestinal stroma, fibroblasts are usually quiescent and are characterized by the expression of both vimentin and desmin. In their inactive state, fibroblasts do not express $\alpha$-smooth muscle actin ( $\alpha$-SMA) but can be stimulated to express $\alpha$-SMA under the influence of the reactive stroma $[7,8,22]$. Activated fibroblasts have large euchromatic nuclei, a prominent nucleoli and, on electron microscopy (EM), prominent rough endoplasmic reticulum (rER) and Golgi apparatus [14, 22]. In cancer research, $\alpha$ $\mathrm{SMA}(+)$ stromal fibroblasts are often referred to as myofibroblasts [7, 8, 14, 22-24]. Some investigators caution against this approach because it tends to label several different cell types "myofibroblasts" despite variable ultrastructural features on EM [22, 25].

Some recommend that the term "myofibroblast" be reserved for cells that satisfy strict criteria:

1. typical appearing, spindle-cell

2. within typical pericellular matrix

3. positive for: $\alpha$-SMA, vimentin, non-muscle myosin and EDA cellular fibronectin.

4. ultrastructural confirmation by EM, demonstrating: prominent rER and Golgi apparatus, myofilaments with focal densities, gap junctions, fibronexuses consisting of converging filaments and external fibronectin fibril [22].

Others endorse a more liberal classification of myofibroblasts, defined by their light microscopic appearance and relative expression of vimentin, desmin and $\alpha$-SMA:

1. V myofibroblasts (vimentin $(+)$ only)

2. VM myofibroblasts (vimentin and myosin $(+)$ )

3. VA myofibroblasts (vimentin and $\alpha-\operatorname{SMA}(+)$ )

4. VAD myofibroblasts (vimentin, $\alpha$-SMA and desmin $(+))[7]$

The clinicopathological correlates of this specific immunohistochemical categorization, however, are uncertain. Given that $\alpha$-SMA is the best immunohistochemical maker of myofibroblasts [26], some advocate classifying only the VA and VAD subtypes as true myofibroblasts [8].

Given the reliance on light microscopy and the utility of immunohistochemistry in cancer research, a reasonable approach is to acknowledge that there is a community of " $\alpha$-SMA(+) fibroblast-like cells", which includes both "physiological cells" as well as "reactive cells". "Physiological" $\alpha-\operatorname{SMA}(+)$ fibroblast-like cells are evident within the normal tissue stroma but "reactive" $\alpha$-SMA $(+)$ fibroblast-like cells are only present within the "reactive" stroma in the setting of tissue injury or disease. $\alpha-\operatorname{SMA}(+)$ 
fibroblast-like "physiological cells" include pancreatic and hepatic stellate cells, pericytes and intestinal subepithelial myofibroblasts (ISEMFs). The $\alpha-\operatorname{SMA}(+)$ fibroblast-like "reactive cells" include the activated stromal fibroblasts seen in benign wound healing as well as the cancer-associated fibroblasts (CAFs) found in the peritumoral stroma [6]. Whilst, all " $\alpha$-SMA $(+)$ fibroblast-like cells" do not conform to the original EM-description of "the myofibroblast" [2729], many of the CAFs do indeed share the ultrastructural features of fully differentiated myofibroblasts [22].

Using the term "myofibroblast" for typical appearing $\alpha$ $\mathrm{SMA}(+)$ cells within the peritumoural stroma, provided they are not peri-endothelial pericytes, is widely endorsed $[5-7,9,11,14,23,26]$. Better still, perhaps, is reserving the term "myofibroblast" for $\alpha-\operatorname{SMA}(+)$ stromal fibroblasts that also have phenotypically consistent expression across additional markers, such as vimentin and prolyl 4hydroxylase $(+)$ and cytokeratin $(-)$ or that are confirmed on EM [30]. In this review, unless satisfying the preceding criteria, fibroblast-like cells within the reactive stroma will be referred to by the less demanding, and less precise [31], term CAF if it is peritumoral or " $\alpha-\operatorname{SMA}(+)$ or $(-)$ stromal fibroblast" if it resides within benign tissue [30]. Unfortunately, such an approach still presents some difficulties. Chronic inflammation often precedes the development of invasive digestive carcinomas. Thus, the same $\alpha-\operatorname{SMA}(+)$ stromal fibroblasts present in the reactive stroma immediately before invasion would be renamed CAFs immediately following invasion, although their structure and function may be unchanged. This issue is particularly important in animal cancer models, such as gastric carcinoma, that have well established, progressive premalignant epithelial pathology [32]. Another difficulty is that CAFs are biologically heterogeneous and may not be adequately categorized by the expression of $\alpha$-SMA alone [31, 33]. Ultimately, more sophisticated gene expression and epigenetic profiling of these cells may resolve some of these uncertainties, particularly if specific molecular signatures can be found that are associated with key outcomes in terms of chronic inflammation and healing [34, 35], or carcinogenesis, metastasis and prognosis [36-38]. Although a combined EM and immunohistochemistry approach is currently the ideal [25], researchers will continue to balance available resources against the benefits of phenotypic and semantic precision.

\section{Other Important Stromal Cells}

\section{Inflammatory Cells}

Inflammatory cells help to define the reactive stroma [32, 39]. In the reactive stroma that accompanies Helicobacter pylori infection and gastritis, there is a vigorous cytokine response [40, 41]. Firstly, neutrophils are recruited to the stroma by IL-8 and then additional chemokines attract chronic inflammatory cells, such as lymphocytes, monocytes and plasma cells [32, 40, 41]. Macrophages differentiate from circulating monocytes under the influence of IL-6 and leukemia inhibitory factor and help to coordinate the inflammatory response [8]. Inflammatory cells are readily discriminated from fibroblasts in the stroma by morphology as well as their expression of the panhematopoietic marker CD45.

\section{Pericytes and Endothelial Cells}

Neovascularization involves the recruitment and proliferation of pericytes and endothelium and is critical in carcinogenesis. Pericytes are usually crescentic, mesenchymal cells, intimately associated with normal endothelium in the microcirculation [14]. Pericytes usually co-express $\alpha$ SMA and desmin [22], and thus may occasionally be confused with myofibroblasts particularly in the setting of the disordered vasculature that accompanies carcinogenesis. Pericytes are important in regulating endothelial permeability and blood flow. Their exact origin is uncertain, but they may arise from local smooth muscle cells or myofibroblasts or alternatively may develop from bone-marrow derived cells (BMDCs), such as multipotent mesenchymal stromal cells (MSCs) [8]. Endothelial cells, of course, are extremely important in health and disease, particularly cancer. Endothelial cells are characterized by co-expression of CD31, von Willebrand factor and CD34 [22]. In cancer, the endothelium originates in part from circulating bone-marrow derived endothelial progenitor cells [42].

\section{Smooth Muscle Cells}

Stromal smooth muscle cells occur within the parenchyma as well as within the walls of arteries and arterioles. Parenchymal smooth muscle cells have far less intercellular matrix surrounding them than is found in the context of fibroblasts and myofibroblasts within the ECM. Nevertheless, the expression of $\alpha$-SMA in both smooth muscle and myofibroblasts may occasionally cause some difficulties in distinguishing these two cells. Smooth muscle cells, however, can be defined by their co-expression of smoothelin and caldesmon [6].

Other Important Physiological Gastrointestinal $\alpha-\operatorname{SMA}(+)$ Cells

\section{Intestinal Subepithelial Myofibroblasts (ISEMFs)}

Intestinal subepithelial myofibroblasts, also known as pericryptal fibroblasts, are $\alpha$-SMA $(+)$ mesenchymal cells that occur throughout the alimentary tract (Fig. 1). They reside immediately beneath the epithelial basement mem- 
Fig. 1 a Gastric cancer and b rectal adenoma in two female patients following male human allogeneic haematopoietic stem cell transplantation. Using Ychr. CISH and $\alpha$-SMA immunohistochemistry bone marrow derived $\alpha$-SMA $(+)$ gastric CAFs in A and ISEMFs in B (black arrows, reproduced with permission) [53]
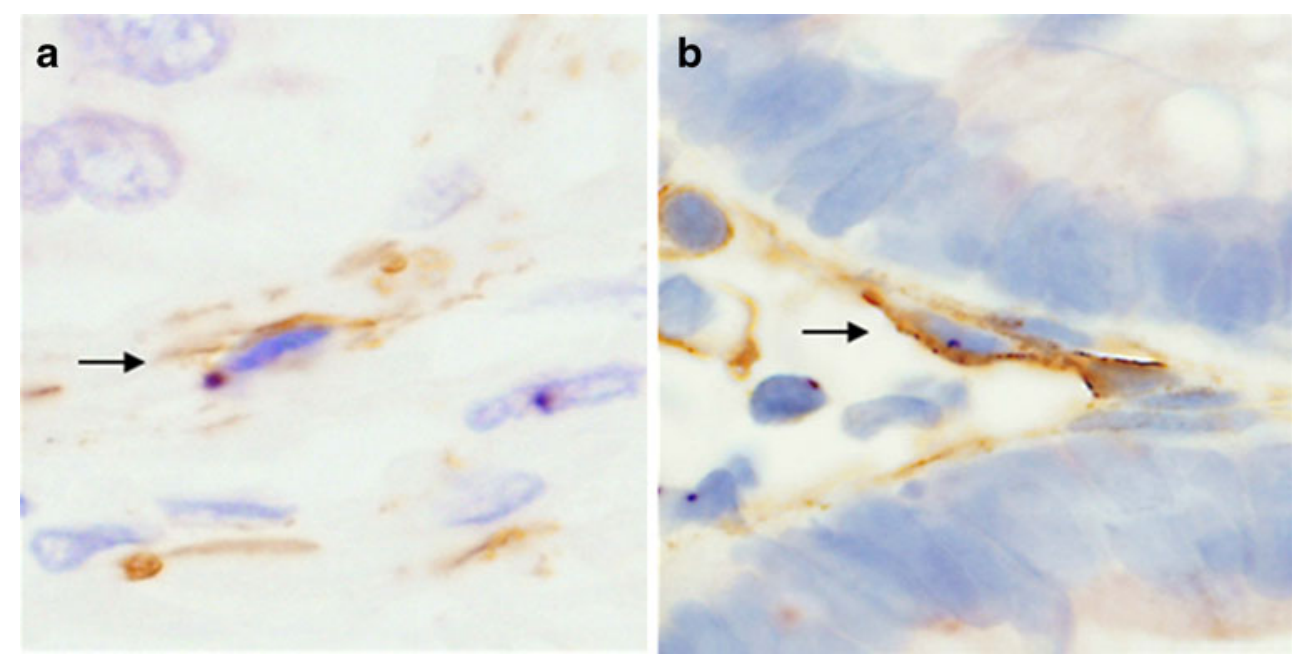

brane [6]. In addition to $\alpha$-SMA, these cells co-express smooth muscle heavy chain myosin, vimentin, the fibroblast markers prolyl 4-hydroxylase and CD90, but not the smooth muscle markers smoothelin or desmin [6]. Furthermore, the smooth muscle marker h-caldesmon has also been identified in some pericryptal cells [22]. These cells interconnect with the other key mesenchymal elements throughout the intestinal lamina propria, including fibroblasts, pericytes and the muscularis mucosae [6]. Electron microscopy suggests that these cells share many of the typical features of myofibroblasts including microfilament bundles, dense bodies and a well developed rER and Golgi apparatus [6]. Typical myofibroblasts, however, tend to have slightly more rER and do not express caldesmon [22].

The putative intestinal "stem cell niche" may be defined by ISEMFs in collaboration with other local cells as well as the basement membrane [6, 7]. The ISEMFs are believed to help create a specialized, discrete microenvironment to nurture, maintain and regulate tissue specific stem cells, to promote their quiescence and capacity for long-term self renewal [43]. In addition, neurons contribute to the melanocyte stem cell niche [44] and have previously been shown to be important within the bone marrow niche [45]. The nervous system might also be important within the development and maintenance of any digestive "stem cell niche".

Intestinal subepithelial myofibroblasts produce regionallyspecific growth factors, possibly contributing to the regional epithelial differences along the alimentary tract and are capable of organizing endoderm into well differentiated villi, containing enterocytes, goblet cells and enteroendocrine cells [6]. Their intestinal differentiating capacity is also evident from studies in which ISEMFs were co-cultured with colon cancer cell lines [46]. T84 cell line colonies, co-cultured with the ISEMFs, organized into basement membrane limited luminal structures and almost half of the colonies developed into single-layer columnar epithelium with intestinal microvilli, alkaline phosphatase, brush border activity and mucin
[46]. The discovery of an intestinal stem cell marker, Lgr5, promised to clarify the nature of the intestinal stem cell niche [47]. Interestingly, however, Lgr5+ intestinal stem cells were recently shown to be capable of semi-autonomous organization into crypt-villus structures, without the need for any discrete cellular mesenchymal niche [48]. In this experiment, however, the supportive environment contained several mesenchymal factors such as EGF and R-spondin 1 [48]. Thus, the precise in vivo role of ISEMFs as possible "niche cells", awaits further research. The exciting corollary of ISEMFs as physiological niche cells is the possible role of some CAFs as pathological niche cells in the setting of digestive cancer stem cells [49]. Furthermore, re-establishing a physiological "niche" might be a possible strategy to restore normal epithelial biology.

\section{Interstitial Cells of Cajal (ICCs)}

ICCs are $\alpha-\operatorname{SMA}(+)$ cells exhibiting typical myofibroblast morphology on light microscopy [23]. On EM, however, these cells appear more like modified smooth muscle cells than typical myofibroblasts [22]. These cells are often found in the intermuscular space between the circular and longitudinal layers of the muscularis propria of the stomach, small intestine and colorectum [23]. ICCs function as pacemaker cells to coordinate smooth muscle motility, facilitate electrical signaling and regulate neurotransmission [23]. ICC dysfunction is implicated in Hirschprung's disease and achalasia and ICCs are the cellular origin of gastrointestinal stromal tumors, the most common abdominal mesenchymal tumor [50].

\section{The Development and Derivation of CAFs and Myofibroblasts}

Gastrointestinal mesenchymal elements begin their development with the encircling of the endoderm by the lateral plate 
mesoderm, to form the visceral mesoderm [51]. The endoderm and mesoderm undergo extensive and integrated development along five different axes: the cranial-caudal, left-right, anteriorposterior, radial and the crypt-villus axes [51]. It is likely that neither the mesoderm nor the endoderm holds all of the information required for gastrointestinal embryogenesis with intimate signaling between these two germ layers being necessary for normal development. It is uncertain whether ISEMFs develop from the mesoderm or the neural crest, but they are evident within the mesenchymal layer by 21 weeks gestation [51]. In mice, ISEMFs have been described from E18.5 (18.5 days post coitum) [51]. ISEMFs are very important in the embryogenesis of the functional epithelial axis throughout the alimentary tract, such as the crypt-villus axis in the small intestine. Hedgehog, Wnt and BMP pathway signaling between the epithelium and the mesoderm, probably via ISEMFs, is critical for normal crypt-villus development [51].

Post-embryogenesis, several animal and human allogeneic stem cell transplantation studies have shown that mesenchymal elements such as ISEMFs and CAFs are derived, at least in part, from BMDCs, possibly MSCs (Figs. 1 and 2) [52-59]. Other studies, however, suggest that local tissue compartments may harbor cells capable of developing into CAFs (Fig. 2) [14, 17, 18].

\section{CAFs and Myofibroblasts in Cancer}

Normal stroma contains very few fibroblasts, but there is a dramatic increase in fibroblast-like cells within the reactive stroma surrounding inflamed or neoplastic tissue [14]. In normal wound healing there is an initial aggregation and activation of platelets, enhanced vascular permeability and an influx of fibrinogen to promote hemostasis. Platelets release a number of regulatory factors including transforming growth factor- $\beta$ (TGF- $\beta$ ) and platelet-derived growth factor (PDGF) leading to an expansion of $\alpha$-SMA $(+)$ fibroblasts and other cells, which in turn produce growth factors, such as vascular-endothelial growth factor (VEGF) and fibroblast growth factor-2 (FGF2)(Fig. 2). $\alpha$-SMA(+) stromal fibroblasts also produce a number of important proteolytic enzymes, which help to generate granulation tissue and achieve re-epithelialization. There are many parallels between normal wound healing and the cancer stroma $[4,59]$.

In inflammatory digestive cancers, such as gastric cancer, the reactive stroma precedes dysplasia and cancer [24]. Similarly, in colorectal adenomas there is already a significant expansion of the ISEMF population compared to normal colorectal mucosa $[60,61]$. This suggests that stromal fibroblasts may play a role in propagating pre-malignant lesions as well as in promoting advanced events such as invasion and metastasis. This notion of an early contribution of stromal fibroblasts to cancer was supported by an elegant study investigating the FSP1-Cre TGF $\beta$ IIR $^{\text {floxfflox }}$ mouse, a model in which all fibroblasts lack the TGF $\beta$ type II receptor $[10,62]$. These mice developed increased rates of forestomach squamous cell carcinoma and prostate neoplasia [62]. This suggested that an underlying abnormality of the fibroblast could initiate as well as advance epithelial cancer $[10,62]$.
Fig. 2 Schematic representation of the gastrointestinal stroma: the key stromal cells and events within the reactive stroma

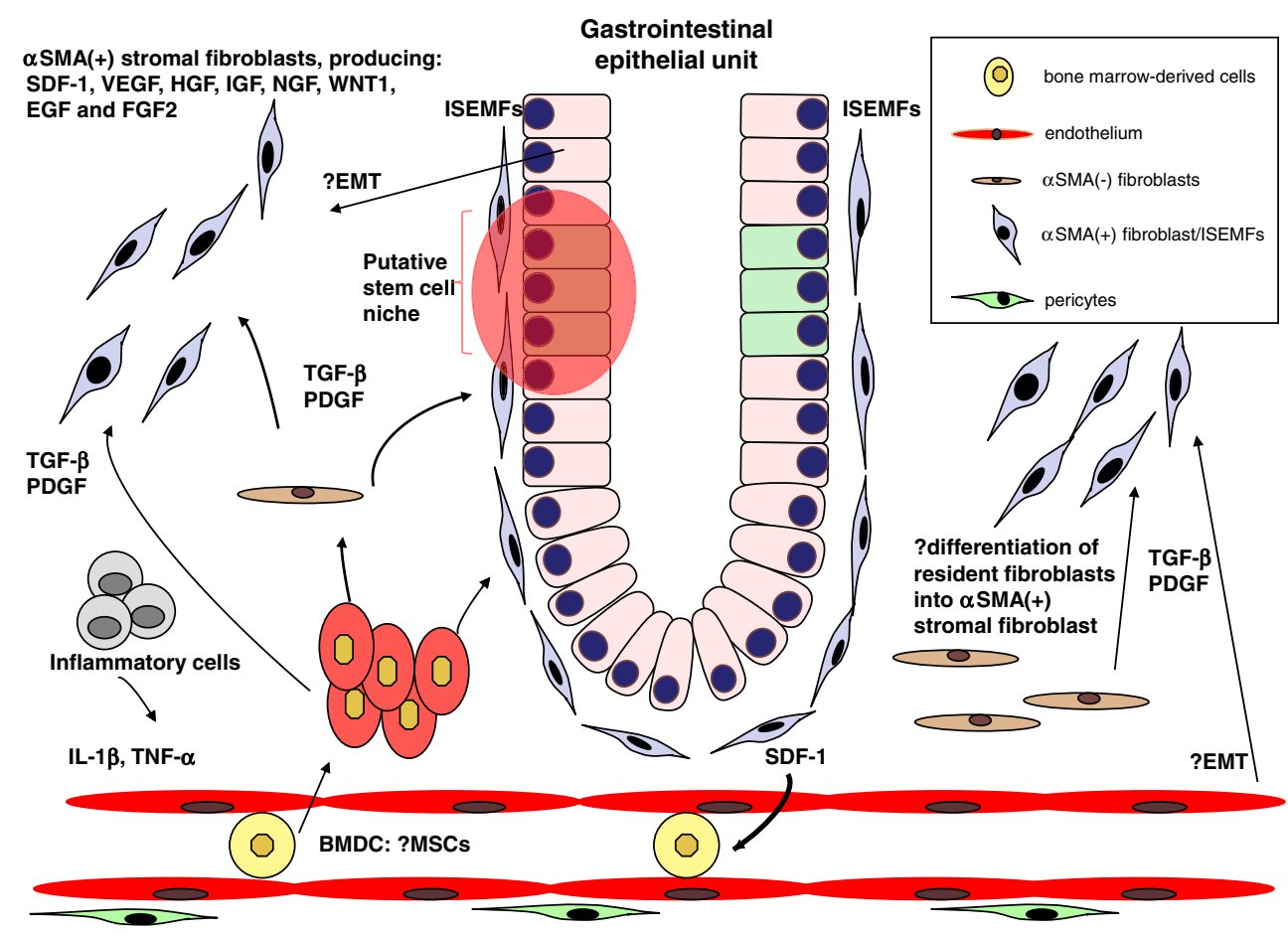


Cancer-associated fibroblasts secrete a number of important inflammatory mediators, including matrixmetalloproteinases $-2,-3$ and -9 , which can alter the stromal ECM and potentiate invasion, cell motility and metastasis [14, 63-66]. Cancer-associated fibroblasts also produce stromal derived factor-1 (SDF-1), VEGF, hepatocyte growth factor (HGF), insulin-like growth factor (IGF), nerve growth factor, WNT1, epidermal growth factor (EGF) and FGF2, which can directly promote growth in the adjacent epithelium and neovascularization within the stroma (Fig. 2) [14]. Stromal fibroblasts may also be important in promoting the seeding and sustenance of distant micrometastasis, possibly mediated through their promotion of local invasion and angiogenesis [14].

A critical issue is whether stromal fibroblasts are normal cells in abnormal circumstances or whether they are different from their normal tissue counterparts. The weight of evidence suggests that CAFs are indeed different to the normal cells $[14,67,68]$. In one study, breast CAFs were more likely than normal fibroblasts to enhance tumor growth, to express $\alpha-\mathrm{SMA}(+)$, to produce $\mathrm{SDF}-1$, to enhance angiogenesis and to stimulate the CXCR4 receptor $[67,68]$. Local SDF-1 production by these cells was also shown to enhance the chemotaxis of CXCR4-expressing endothelial progenitor cells (EPCs) and hematopoietic progenitor cells (HPCs), into the stroma [68]. CXCR4 is also expressed by a small proportion of MSCs [69], which may be relevant in the recruitment of these cells into the stroma to develop into bone marrow derived CAFs. Cancer-associated fibroblasts from prostate cancer are also significantly different, showing greater tumor potentiating effects in vitro and in vivo compared to normal prostatic fibroblasts [70].

The increase in $\alpha$-SMA $(+)$ stromal fibroblasts within the reactive stroma may occur through a combination of different mechanisms. Firstly, chemokines such as SDF-1 may recruit fibroblast precursors, possibly MSCs or HPCs, into the stroma from the bone marrow. In the context of additional factors, including PDGF and TGF- $\beta$, these precursors may differentiate into $\alpha-\operatorname{SMA}(+)$ stromal fibroblasts (Fig. 2). In culture, TGF- $\beta$ can activate normal fibroblasts into $\alpha$-SMA $(+)$ fibroblasts [71]. Secondly, TGF$\beta$ and PDGF may act on resident inactive fibroblasts to induce myofibroblastic differentiation [72]. Finally, epithelial-mesenchymal transition (EMT), possibly also promoted by PDGF and TGF- $\beta$, could explain some of the stromal fibroblasts $[14,17,18]$.

It is likely that CAFs are biologically heterogeneous and that this heterogeneity extends beyond merely whether they do, or do not, express $\alpha$-SMA $[31,33]$. One study recently characterized stromal fibroblasts from two mouse models of cancer [33]. They identified a distinct subpopulation of FSP-1(+) CAFs that did not seem to significantly express other "typical" stromal fibroblast markers, such as $\alpha$-SMA, vimentin, fibroblast-activation protein, fibroblast-associated antigen or prolyl 4-hydroxylase [33]. This study suggested a biological dichotomy within stromal fibroblasts. This could reflect the activity of the cells studied, activated versus nonactivated, or perhaps even the origin of the cells, local as opposed to bone marrow derived. New markers and new techniques may be needed to understand the heterogeneous biology and thus diverse stromal consequences of CAFs.

Gastric cancer is one of the key digestive cancer models that has explored the origin and the role of CAFs $[8,24,32$, 39, 53, 54, 58, 73, 74]. From studies using the Helicobacter felis $\mathrm{C} 57 \mathrm{BL} / 6$ model of gastric cancer, it is clear that bonemarrow derived $\alpha-\operatorname{SMA}(+)$ fibroblasts enter the premalignant chronically inflamed reactive gastric stroma [54]. Bone marrow derived CAFs have also been confirmed in human gastric cancer (Fig. 1a) [53]. As discussed above, these cells are likely to play an important role in the progression and potentially even in the initiation of gastric carcinogenesis [10, 62].

\section{Are CAFs Molecularly Transformed?}

Excluding rare examples of primary myofibroblastic malignancies [75], CAF precursors presumably begin as normal cells [68]. A possible caveat, outlined above, would be if CAFs developed via EMT from neoplastic epithelium. In this scenario, one would expect to find common genetic abnormalities in epithelium and stroma. Once activated, however, CAFs seem to maintain their activated phenotype even when removed from the activated stroma [68]. This led researchers to consider whether these cells had undergone any genetic alterations that would sustain their autonomous activity. The results have been somewhat mixed [68]. In 2002, an exciting report emerged that mutations and loss of heterozygosity at TP53 and PTEN could be detected in breast cancer stroma [76, 77]. Although the genetic aberrations in cancer and stroma were usually discordant, there were 2 cases of identical mutations in TP53 [77]. This report was followed by a series of informative studies conducted in patients with inherited as well as sporadic breast cancer [78-80]. In all studies they confirmed the presence of significant stromal genetic instability [7880]. Furthermore, in one study they found that stromal LOH was closely correlated with important clinicopathological characteristics, including tumor grade and stage [80].

These findings of stromal genetic instability were supported by a second group studying ovarian cancer [81, 82]. These studies initially analyzed 6 microsatellites on chromosome $3 p$ [82], and then confirmed the genetic instability in a larger study at 110 loci [81]. Interestingly, they described a number of genetic events that were common to cancer and stroma, raising the possibility of 
EMT [81, 82]. The presence of stromal genetic instability has also been described in colitis-associated colorectal cancer and in a mouse model of prostate cancer [83, 84].

Not all studies, however, have identified significant genetic alterations in the stroma $[68,85,86]$. One recent study analyzed 25 ovarian and 10 breast cancer specimens using a 500K SNP array platform [86]. Only one patient was identified with LOH in the stromal compartment [86]. Reconciling these discrepant results is difficult owing to the inherent differences in the types of tissues analyzed, fresh frozen vs. archival formalin fixed specimens, the different techniques used and the exact compartments, and thus cells, analyzed [86].

There is greater consensus in support of epigenetic changes in CAFs [74, 87, 88]. One study analyzed promoter region methylation in the stroma of five patients with prostate cancer [87]. Aberrant stromal methylation was reported in four patients, but it was unclear whether these changes were truly stroma-specific [87]. The other studies were more persuasive and adopted a genome-wide approach to DNA methylation [74, 88]. The first, used methylation-specific digital karyotyping to analyze DNA methylation in breast cancer cells, associated myoepithelial cells and stromal fibroblasts [88]. Distinct epigenetic changes were found for each cell type [88]. The second study, more interesting from a digestive cancer perspective, used methylation-sensitive SNP arrays to analyze DNA methylation in gastric CAFs [74]. The gastric CAFs had widespread DNA hypomethylation, which was confirmed by loss of 5-methylcytosine staining on immunohistochemistry [74]. DNA hypomethylation is a frequent event in cancer [89]. DNA hypomethylation of repetitive genomic sequences in cancer is associated with genetic instability [89]. It is possible that DNA hypomethylation in CAFs also promotes genetic instability.

These studies suggest that genetic abnormalities can occur within the stroma [20], although the frequency is uncertain and it may be relatively uncommon [86, 90]. It is likely, however, that CAFs have an altered epigenetic profile, which may help to explain their unique biology [90]. Future studies should focus on cell specific rather than compartmental analysis to help resolve some of the discrepancies and better characterize the evolution and biology of these important cells.

\section{Clinical Potential of Stromal Fibroblast Research in Digestive Cancer}

There are several exciting potential clinical implications of stromal fibroblast research for the prevention, diagnosis and management of digestive cancer. The accessibility of the digestive tract to endoscopic sampling provides the opportunity of integrating stromal fibroblast analysis into digestive cancer surveillance for conditions such as Barrett's esophagus or chronic ulcerative colitis. For patients with cancer, new agents could be developed to antagonize the CAFrelated promotion of tissue invasion and metastasis. To some extent this has already been realized with the development of bevacizumab, which is an anti-VEGF monoclonal antibody used in the treatment of several solid organ cancers, including colorectal cancer. Furthermore, the tumor stroma could be manipulated to improve the delivery of chemotherapy. An inhibitor of hedgehog signaling was recently used in an animal model of pancreatic cancer. The agent inhibited peritumoral stromal desmoplasia and as a result improved drug delivery to the tumor [91]. Finally, given that CAFs can originate from the bone marrow, a therapeutically manipulable compartment, CAF-related research could theoretically prefigure attempts to use stem cell transplantation as a means of indirectly seeding and thus modifying the cancer microenvironment.

\section{Summary}

Stromal fibroblasts are critical in the development of digestive cancer. The reactive, peritumoral stroma shares many characteristics with the reactive stroma associated with "normal" wound healing [4]. Expression of $\alpha$-SMA in stromal fibroblasts is a good marker of myofibroblastic differentiation, but may not adequately categorize the full biological heterogeneity of CAFs. In the future, expression profiling or other means of genetic and epigenetic analysis may help to better characterize these cells into coherent subtypes. In turn, improved characterization may facilitate a better appreciation of the dynamic changes that occur within the stroma. Understanding the origin and biology of stromal fibroblasts will help in the development of an integrated epithelialstromal sequence in digestive cancers and will ultimately inform pathogenesis, natural history and future therapeutics.

Open Access This article is distributed under the terms of the Creative Commons Attribution Noncommercial License which permits any noncommercial use, distribution, and reproduction in any medium, provided the original author(s) and source are credited.

\section{References}

1. Parkin DM (2001) Global cancer statistics in the year 2000 . Lancet Oncol 2:533-543

2. Jemal A, Siegel R, Ward E et al (2008) Cancer statistics, 2008. CA Cancer J Clin 58:71-96

3. Bissell MJ, Radisky D (2001) Putting tumours in context. Nat Rev Cancer 1:46-54

4. Dvorak HF (1986) Tumors: wounds that do not heal. Similarities between tumor stroma generation and wound healing. N Engl J Med 315:1650-1659 
5. Liotta LA, Kohn EC (2001) The microenvironment of the tumourhost interface. Nature 411:375-379

6. Powell DW, Adegboyega PA, Di Mari JF et al (2005) Epithelial cells and their neighbors I. Role of intestinal myofibroblasts in development, repair, and cancer. Am J Physiol Gastrointest Liver Physiol 289:G2-G7

7. Powell DW, Mifflin RC, Valentich JD et al (1999) Myofibroblasts. I. Paracrine cells important in health and disease. Am J Physiol 277:C1-C9

8. Varro A (2009) Stromal cells and the tumor microenvironment. In: Wang TC, Fox JG, Giraud AS (eds) The biology of gastric cancers, 1st edn. Springer, New York, pp 587-600

9. Radisky D, Muschler J, Bissell MJ (2002) Order and disorder: the role of extracellular matrix in epithelial cancer. Cancer Invest 20:139-153

10. Bhowmick NA, Neilson EG, Moses HL (2004) Stromal fibroblasts in cancer initiation and progression. Nature 432:332-337

11. Kalluri R (2003) Basement membranes: structure, assembly and role in tumour angiogenesis. Nat Rev Cancer 3:422-433

12. Simon-Assmann P, Kedinger M, De Arcangelis A et al (1995) Extracellular matrix components in intestinal development. Experientia 51:883-900

13. Schuppan D, Schmid M, Somasundaram R et al (1998) Collagens in the liver extracellular matrix bind hepatocyte growth factor. Gastroenterology 114:139-152

14. Kalluri R, Zeisberg M (2006) Fibroblasts in cancer. Nat Rev Cancer 6:392-401

15. Gaggioli C, Hooper S, Hidalgo-Carcedo C et al (2007) Fibroblastled collective invasion of carcinoma cells with differing roles for RhoGTPases in leading and following cells. Nat Cell Biol 9:1392-1400

16. Hanahan D, Weinberg RA (2000) The hallmarks of cancer. Cell 100:57-70

17. Zeisberg EM, Potenta S, Xie L et al (2007) Discovery of endothelial to mesenchymal transition as a source for carcinomaassociated fibroblasts. Cancer Res 67:10123-10128

18. Zeisberg M, Yang C, Martino $M$ et al (2007) Fibroblasts derive from hepatocytes in liver fibrosis via epithelial to mesenchymal transition. J Biol Chem 282:23337-23347

19. Paget $S$ (1889) The distribution of secondary growths in cancer of the breast. Lancet 1:571-573

20. Eng C, Leone G, Orloff MS et al (2009) Genomic alterations in tumor stroma. Cancer Res 69:6759-6764

21. Umar A, Boland CR, Terdiman JP et al (2004) Revised Bethesda guidelines for hereditary nonpolyposis colorectal cancer (Lynch syndrome) and microsatellite instability. J Natl Cancer Inst 96:261-268

22. Eyden B (2005) The myofibroblast: a study of normal, reactive and neoplastic tissues, with an emphasis on ultrastructure. Part 1 normal and reactive cells. J Submicrosc Cytol Pathol 37:109-204

23. Powell DW, Mifflin RC, Valentich JD et al (1999) Myofibroblasts. II. Intestinal subepithelial myofibroblasts. Am J Physiol 277:C183-C201

24. McCaig C, Duval C, Hemers E et al (2006) The role of matrix metalloproteinase-7 in redefining the gastric microenvironment in response to Helicobacter pylori. Gastroenterology 130:1754-1763

25. Eyden B (2009) The myofibroblast, electron microscopy and cancer research. Int J Cancer 125:1743-1745, author reply 1746

26. Gabbiani G (2003) The myofibroblast in wound healing and fibrocontractive diseases. J Pathol 200:500-503

27. Majno G, Gabbiani G, Hirschel BJ et al (1971) Contraction of granulation tissue in vitro: similarity to smooth muscle. Science 173:548-550

28. Ryan GB, Cliff WJ, Gabbiani G et al (1974) Myofibroblasts in human granulation tissue. Hum Pathol 5:55-67

29. Gabbiani G, Ryan GB, Majne G (1971) Presence of modified fibroblasts in granulation tissue and their possible role in wound contraction. Experientia 27:549-550
30. De Wever O, Demetter P, Mareel M et al (2008) Stromal myofibroblasts are drivers of invasive cancer growth. Int J Cancer 123:2229-2238

31. Orimo A, Weinberg RA (2007) Heterogeneity of stromal fibroblasts in tumors. Cancer Biol Ther 6:618-619

32. Correa P, Houghton J (2007) Carcinogenesis of helicobacter pylori. Gastroenterology 133:659-672

33. Sugimoto H, Mundel TM, Kieran MW et al (2006) Identification of fibroblast heterogeneity in the tumor microenvironment. Cancer Biol Ther 5:1640-1646

34. Wall IB, Moseley R, Baird DM et al (2008) Fibroblast dysfunction is a key factor in the non-healing of chronic venous leg ulcers. J Invest Dermatol 128:2526-2540

35. Stanczyk J, Pedrioli DM, Brentano F et al (2008) Altered expression of MicroRNA in synovial fibroblasts and synovial tissue in rheumatoid arthritis. Arthritis Rheum 58:1001-1009

36. Singer CF, Gschwantler-Kaulich D, Fink-Retter A et al (2008) Differential gene expression profile in breast cancer-derived stromal fibroblasts. Breast Cancer Res Treat 110:273-281

37. Verona EV, Elkahloun AG, Yang J et al (2007) Transforming growth factor-beta signaling in prostate stromal cells supports prostate carcinoma growth by up-regulating stromal genes related to tissue remodeling. Cancer Res 67:5737-5746

38. Chang HY, Nuyten DS, Sneddon JB et al (2005) Robustness, scalability, and integration of a wound-response gene expression signature in predicting breast cancer survival. Proc Natl Acad Sci U S A 102:3738-3743

39. Houghton J, Wang TC (2005) Helicobacter pylori and gastric cancer: a new paradigm for inflammation-associated epithelial cancers. Gastroenterology 128:1567-1578

40. Fox JG, Wang TC (2007) Inflammation, atrophy, and gastric cancer. J Clin Invest 117:60-69

41. D'Elios MM, Andersen LP (2009) Inflammation, immunity, and vaccines for helicobacter pylori. Helicobacter 14(Suppl 1):21-28

42. Peters BA, Diaz LA, Polyak K et al (2005) Contribution of bone marrow-derived endothelial cells to human tumor vasculature. Nat Med 11:261-262

43. Yen TH, Wright NA (2006) The gastrointestinal tract stem cell niche. Stem Cell Rev 2:203-212

44. Adameyko I, Lallemend F, Aquino JB et al (2009) Schwann cell precursors from nerve innervation are a cellular origin of melanocytes in skin. Cell 139:366-379

45. Mendez-Ferrer S, Lucas D, Battista M et al (2008) Haematopoietic stem cell release is regulated by circadian oscillations. Nature 452:442-447

46. Halttunen T, Marttinen A, Rantala I et al (1996) Fibroblasts and transforming growth factor beta induce organization and differentiation of T84 human epithelial cells. Gastroenterology 111:1252-1262

47. Barker N, van Es JH, Kuipers J et al (2007) Identification of stem cells in small intestine and colon by marker gene Lgr5. Nature 449:1003-1007

48. Sato T, Vries RG, Snippert HJ et al (2009) Single Lgr5 stem cells build crypt-villus structures in vitro without a mesenchymal niche. Nature 459:262-265

49. Malanchi I, Huelsken J (2009) Cancer stem cells: never Wnt away from the niche. Curr Opin Oncol 21:41-46. doi:10.1097/CCO.1090 b1013e32831d32831faf

50. Coffey RJ, Washington MK, Corless CL et al (2007) Menetrier disease and gastrointestinal stromal tumors: hyperproliferative disorders of the stomach. J Clin Invest 117:70-80

51. McLin VA, Henning SJ, Jamrich M (2009) The role of the visceral mesoderm in the development of the gastrointestinal tract. Gastroenterology 136:2074-2091

52. Brittan M, Hunt T, Jeffery R et al (2002) Bone marrow derivation of pericryptal myofibroblasts in the mouse and human small intestine and colon. Gut 50:752-757 
53. Worthley DL, Ruszkiewicz A, Davies R et al (2009) Human gastrointestinal neoplasia-associated myofibroblasts can develop from bone marrow-derived cells following allogeneic stem cell transplantation. Stem Cells 27:1463-1468

54. Houghton J, Stoicov C, Nomura S et al (2004) Gastric cancer originating from bone marrow-derived cells. Science 306:1568-1571

55. Marrache F, Pendyala S, Bhagat G et al (2008) Role of bone marrowderived cells in experimental chronic pancreatitis. Gut 57:1113-1120

56. Direkze NC, Hodivala-Dilke K, Jeffery R et al (2004) Bone marrow contribution to tumor-associated myofibroblasts and fibroblasts. Cancer Res 64:8492-8495

57. Mishra PJ, Mishra PJ, Humeniuk R et al (2008) Carcinomaassociated fibroblast-like differentiation of human mesenchymal stem cells. Cancer Res 68:4331-4339

58. Guo X, Oshima H, Kitmura T et al (2008) Stromal fibroblasts activated by tumor cells promote angiogenesis in mouse gastric cancer. J Biol Chem 283:19864-19871

59. Stappenbeck TS, Miyoshi H (2009) The role of stromal stem cells in tissue regeneration and wound repair. Science 324:1666-1669

60. Adegboyega PA, Mifflin RC, DiMari JF et al (2002) Immunohistochemical study of myofibroblasts in normal colonic mucosa, hyperplastic polyps, and adenomatous colorectal polyps. Arch Pathol Lab Med 126:829-836

61. Adegboyega PA, Ololade O, Saada J et al (2004) Subepithelial myofibroblasts express cyclooxygenase- 2 in colorectal tubular adenomas. Clin Cancer Res 10:5870-5879

62. Bhowmick NA, Chytil A, Plieth D et al (2004) TGF-beta signaling in fibroblasts modulates the oncogenic potential of adjacent epithelia. Science 303:848-851

63. Lochter A, Galosy S, Muschler J et al (1997) Matrix metalloproteinase stromelysin-1 triggers a cascade of molecular alterations that leads to stable epithelial-to-mesenchymal conversion and a premalignant phenotype in mammary epithelial cells. J Cell Biol 139:1861-1872

64. Lochter A, Sternlicht MD, Werb Z et al (1998) The significance of matrix metalloproteinases during early stages of tumor progression. Ann N Y Acad Sci 857:180-193

65. Lochter A, Srebrow A, Sympson CJ et al (1997) Misregulation of stromelysin-1 expression in mouse mammary tumor cells accompanies acquisition of stromelysin-1-dependent invasive properties. J Biol Chem 272:5007-5015

66. Sternlicht MD, Lochter A, Sympson CJ et al (1999) The stromal proteinase MMP3/stromelysin-1 promotes mammary carcinogenesis. Cell 98:137-146

67. Orimo A, Gupta PB, Sgroi DC et al (2005) Stromal fibroblasts present in invasive human breast carcinomas promote tumor growth and angiogenesis through elevated SDF-1/CXCL12 secretion. Cell 121:335-348

68. Orimo A, Weinberg RA (2006) Stromal fibroblasts in cancer: a novel tumor-promoting cell type. Cell Cycle 5:1597-1601

69. Wynn RF, Hart CA, Corradi-Perini C et al (2004) A small proportion of mesenchymal stem cells strongly expresses functionally active CXCR4 receptor capable of promoting migration to bone marrow. Blood 104:2643-2645

70. Olumi AF, Grossfeld GD, Hayward SW et al (1999) Carcinomaassociated fibroblasts direct tumor progression of initiated human prostatic epithelium. Cancer Res 59:5002-5011

71. Ronnov-Jessen L, Petersen OW (1993) Induction of alpha-smooth muscle actin by transforming growth factor-beta 1 in quiescent human breast gland fibroblasts. Implications for myofibroblast generation in breast neoplasia. Lab Invest 68:696-707

72. Ronnov-Jessen L, Petersen OW, Koteliansky VE et al (1995) The origin of the myofibroblasts in breast cancer. Recapitulation of tumor environment in culture unravels diversity and implicates converted fibroblasts and recruited smooth muscle cells. J Clin Invest 95:859-873

73. Quante M, Wang TC (2008) Inflammation and stem cells in gastrointestinal carcinogenesis. Physiology (Bethesda) 23:350 359

74. Jiang L, Gonda TA, Gamble MV et al (2008) Global hypomethylation of genomic DNA in cancer-associated myofibroblasts. Cancer Res 68:9900-9908

75. Eyden B (2005) The myofibroblast: a study of normal, reactive and neoplastic tissues, with an emphasis on ultrastructure. part 2tumours and tumour-like lesions. J Submicrosc Cytol Pathol 37:231296

76. Loh K, Chia JA, Greco S et al (2008) Bone morphogenic protein 3 inactivation is an early and frequent event in colorectal cancer development. Genes, chromosomes cancer 47(6):449-460

77. Kurose K, Gilley K, Matsumoto S et al (2002) Frequent somatic mutations in PTEN and TP53 are mutually exclusive in the stroma of breast carcinomas. Nat Genet 32:355-357

78. Weber F, Shen L, Fukino K et al (2006) Total-genome analysis of BRCA1/2-related invasive carcinomas of the breast identifies tumor stroma as potential landscaper for neoplastic initiation. Am J Hum Genet 78:961-972

79. Fukino K, Shen L, Matsumoto S et al (2004) Combined total genome loss of heterozygosity scan of breast cancer stroma and epithelium reveals multiplicity of stromal targets. Cancer Res 64:7231-7236

80. Fukino K, Shen L, Patocs A et al (2007) Genomic instability within tumor stroma and clinicopathological characteristics of sporadic primary invasive breast carcinoma. JAMA 297:21032111

81. Tuhkanen H, Anttila M, Kosma VM et al (2006) Frequent gene dosage alterations in stromal cells of epithelial ovarian carcinomas. Int J Cancer 119:1345-1353

82. Tuhkanen H, Anttila M, Kosma VM et al (2004) Genetic alterations in the peritumoral stromal cells of malignant and borderline epithelial ovarian tumors as indicated by allelic imbalance on chromosome 3p. Int J Cancer 109:247-252

83. Hill R, Song Y, Cardiff RD et al (2005) Selective evolution of stromal mesenchyme with p53 loss in response to epithelial tumorigenesis. Cell 123:1001-1011

84. Matsumoto N, Yoshida T, Okayasu I (2003) High epithelial and stromal genetic instability of chromosome 17 in ulcerative colitisassociated carcinogenesis. Cancer Res 63:6158-6161

85. Allinen M, Beroukhim R, Cai L et al (2004) Molecular characterization of the tumor microenvironment in breast cancer. Cancer Cell 6:17-32

86. Qiu W, Hu M, Sridhar A et al (2008) No evidence of clonal somatic genetic alterations in cancer-associated fibroblasts from human breast and ovarian carcinomas. Nat Genet 40:650-655

87. Hanson JA, Gillespie JW, Grover A et al (2006) Gene promoter methylation in prostate tumor-associated stromal cells. J Natl Cancer Inst 98:255-261

88. Hu M, Yao J, Cai L et al (2005) Distinct epigenetic changes in the stromal cells of breast cancers. Nat Genet 37:899-905

89. Esteller M (2008) Epigenetics in cancer. N Engl J Med 358:11481159

90. Campbell I, Polyak K, Haviv I (2009) Clonal mutations in the cancer-associated fibroblasts: the case against genetic coevolution. Cancer Res 69:6765-6768, discussion 6769

91. Olive KP, Jacobetz MA, Davidson CJ et al (2009) Inhibition of hedgehog signaling enhances delivery of chemotherapy in a mouse model of pancreatic cancer. Science 324:1457-1461 